\title{
Hematopoietic Stem Cell Transplantation in CARD9 Deficiency: Knight in Shining Armor?
}

\author{
Isabelle Meyts ${ }^{1,2,3}$ iD \\ Received: 7 June 2019 / Accepted: 11 June 2019 / Published online: 19 June 2019 \\ (C) Springer Science+Business Media, LLC, part of Springer Nature 2019
}

In this Issue of the Journal of Clinical Immunology, QueirozTelles et al. report the first successful use of hematopoietic stem cell transplantation (HSCT) in two unrelated patients with autosomal recessive Caspase Recruitment Domain 9 (CARD9) deficiency suffering from recurrent and ongoing invasive dermatophytosis [1]. Autosomal recessive (CARD9) deficiency (OMIM 212050) has recently been extensively reviewed in this journal [2]. In brief, the condition underlies life-threatening invasive infections by ascomycete fungi typically including invasive and superficial dermatophytosis, invasive phaeohyphomycosis, but also invasive Candida (especially central nervous system infection), and extrapulmonary aspergillosis, in otherwise healthy individuals. Since the first description in 2009, 58 patients from 39 kindreds have been reported with homozygous ( $n=49 ; 31$ kindreds) or compound heterozygous ( $n=9 ; 8$ kindreds) CARD9 mutations. The clinical penetrance of fungal disease is complete in all patients described until now, albeit only at age 52 years.

The expression pattern of CARD9 has been incompletely studied. It is however expressed in monocytes and engages in the B cell lymphoma (BCL)-CARD-mucosa-associated lymphoid tissue lymphoma (MALT)-signaling complex downstream of C-type lectin receptors leading to activation of canonical NF-kB and mitogen-activated protein kinase (MAPK) pathway in response to fungal components. Pro-inflammatory cytokine and chemokine responses to various fungal ligands

Isabelle Meyts

Isabelle.Meyts@uzleuven.be

1 UZ Leuven, Department of Pediatrics, University Hospitals Leuven, Herestraat 49, Leuven, Belgium

2 Laboratory for Inborn Errors of Immunity, Department of Immunology, Microbiology and Transplantation, KU Leuven, Leuven, Belgium

3 Precision Immunology Institute and Mindich Child Health and Development Institute, Icahn School of Medicine at Mount Sinai, New York City, NY, USA are impaired in CARD9-deficient patients' peripheral blood mononuclear cells (PBMCs), monocytes, monocyte-derived macrophages, and monocyte-derived dendritic cells, and impaired Th17 immunity was reported in some but not all studied patients [2]. In two patients with invasive extrapulmonary aspergillosis, CARD9-deficient neutrophils killed Aspergillus effectively but did not accumulate at sites of infection, parallel to observations made in Candida infection of the central nervous system [3]. Nevertheless, the precise mechanism of susceptibility to invasive fungal infections (IFI) in patients with CARD9 deficiency remains to be defined.

The main focus of treatment in CARD9 deficiency lies in effective antifungal therapy. Obviously, the choice of treatment depends on the fungal infection observed; the type of infection (mucocutaneous versus invasive); the fungal susceptibility pattern in vitro; the initial clinical, biochemical, and radiological response; and the overall tolerance to the treatment. Caution is needed for relapses under treatment as well as for recurrence of infection [2]. As a rule, fungal infection in CARD9 deficiency requires prolonged treatment with one or more antifungal agents and secondary prophylaxis is a safe option. Moreover, extensive or deep dermatophytosis and fungal abscesses or masses require surgical debridement.

Besides antifungal therapy, growth factor therapy may be utilized. Indeed, in five patients studied, a reduced production of granulocyte macrophage colony-stimulating factor (GMCSF) by PBMCs and monocytes in response to stimulation with zymosan or C. albicans was observed [4-6]. Hence, GCSF and GM-CSF therapy were used in three patients with Candida central nervous system (CNS) infection adjacent to voriconazole treatment with good result $[4,6]$. However, in one patient with recurrent Candida CNS infection, GM-CSF therapy led to eosinophil-driven inflammation in the cerebrospinal fluid and the brain [7]. In analogy to chronic granulomatous disease, IFN-gamma has been used as adjunctive therapy in CARD9 deficiency, however, without success [1]. Despite long-term antifungal therapy and primary and secondary anti-fungal prophylaxes, most patients relapse $(2 / 3)$, 
disseminated disease is common (32\% of patients) and mortality is significant with 10 of 58 patients $(17.2 \%)$ succumbing to active fungal infection at a mean age of 23.1 years (range (12-39 years)). Moreover, the invasive and mutilating infections, often necessitating amputations, impose an important morbidity burden upon affected patients.

At first sight, given this important mortality and morbidity and given the data on impaired inflammatory responses in hematopoietic myeloid-derived as well as on low Th17 cells, the use of HSCT as a definitive treatment may look obvious. However, the lack of data on the expression and role of CARD9 in for instance tissue resident macrophages, which are not hematopoietic stem cell-derived, and in epithelial cells, pose reasonable doubt on HSCT as a treatment option. Previously, HSCT had been undertaken in a 13-year-old boy suffering from ongoing extrapulmonary (intra-abdominal) invasive aspergillosis despite surgical debulking and aggressive antifungal therapy [3]. He received a double-unrelated cord blood (UCB) graft following fludarabine/busulfan/cyclophosphamide/anti-thymocyte globulin (ATG) but experienced primary graft failure (personal communication, Dr. Vinod K. Prasad, Pediatric Blood and Marrow Transplantation, Duke University, Durham, USA). A second UCB graft was performed after conditioning with fludarabine/cyclophosphamide/equine ATG and total body irradiation (TBI) $(2 \times$ $200 \mathrm{cGy}$ ) but the patient succumbed to severe venoocclusive disease and alveolar hemorrhage 8 weeks later [3].

In this issue, successful HSCT is reported in two patients with early onset superficial and deep dermatophytosis and ongoing infection when going into HSCT. Both patients received HSCT from a healthy sibling donor who was heterozygous for the pathogenic mutation. Conditioning regimens were a busulfan/fludarabine/ATG protocol for the patient receiving non-T cell-depleted bone marrow from a matched sibling donor and a fludarabine/cyclophosphamide/TBI and post-transplant cyclophosphamide protocol in the patient receiving T-replete peripheral blood stem cells from a haploidentical sibling. Neutrophil engraftment was swift in both patients ( $d+16$ and $d+18$, respectively). $P 1$ received granulocyte transfusions on D6-D10-D11. Both patients were able to stop anti-fungal treatment, at $\mathrm{d}+100$ and $\mathrm{d}+218$ post-HSCT respectively and are alive and well at 42 and $36 \mathrm{~m}$ post-HSCT. Last obtained chimerism showed full donor chimerism at $\mathrm{d}+$ 360 and at $\mathrm{d}+1000$, respectively. Unfortunately, one patient had to undergo bilateral lower leg amputation. The other patient presented lymphadenopathy at $d+900$ with evidence of fungus infection yet with no fungal growth from the node.

We prudently welcome the current report as a green light for considering HSCT in CARD9-deficiency patients with extensive, poorly responsive, or relapsing fungal infection. Indeed, HSCT has been successful in other primary immunodeficiency disorders characterized by a myeloid cell defect and predisposing to recurrent and invasive fungal infection, such as GATA2 deficiency or chronic granulomatous disease $[8,9]$. In the latter condition, the "old" approach of reserving HSCT for the severely affected (young) patient has shifted to early HSCT in children and HSCT in severely ill adult patients, thanks to the introduction of lower toxicity conditioning regimens resulting in excellent survival (>90\%) with sufficient myeloid donor chimerism. Moreover, a single-center survey showed that the quality of life of the transplanted patients was superior to that of the non-transplanted patients [10]. However, it is prudent not to extrapolate enthusiastically the here reported success in two patients with invasive dermatophytosis, to patients with invasive aspergillosis or mucorales infection. As for optimal conditioning regimen, a reduced busulfan or treosulfan/fludarabine regimen with serotherapy and a T-replete fludarabine/cyclophosphamide protocol in haploidentical setting proved adequate, although the need for TBI may be questioned. It seems wise to avoid UCB donors and to opt for a haploidentical donor if no MUD or MSD is available for swift myeloid engraftment. It is unknown what expression level of CARD9 is required for disease-free survival. In the reported cases, the donors where heterozygous for the pathogenic mutation, which may be problematic in case of incomplete donor chimerism. If the luxury of choice occurs, a MRD not carrying a CARD9 mutation should be chosen.

Last but not least, this report is also important from another perspective. There is reasonable doubt that non-hematopoietic cells play a role in the phenotype observed in CARD9 deficiency. Successful HSCT thus also pleas for a major role of the hematopoietic progenitor cell-derived myeloid cells in the pathogenesis of fungal infections in CARD9 deficiency. The role of tissue-resident macrophages, however, needs further investigation. Optimistically, it can be hypothesized that, as was the case with chronic granulomatous disease, as experience builds, the indication for HSCT in CARD9 deficiency may move from the most severely affected patient with active infection to the less severely affected patient prior to the onset of debilitating chronic fungal infection.

\section{Compliance with Ethical Standards}

Conflict of Interest The author declares she has no conflict of interest.

\section{References}

1. Queiroz-Telles F, Mercier T, Maertens J, Sola CBS, Bonfim C, Lortholary O, et al. Successful allogenic stem cell transplantation in patients with inherited CARD9 deficiency. J Clin Immunol. 2019;39(5). https://doi.org/10.1007/s10875-019-00662-z.

2. Corvilain E, Casanova JL, Puel A. Inherited CARD9 deficiency: invasive disease caused by ascomycete fungi in previously healthy children and adults. J Clin Immunol. 2018;38(6):656-93. 
3. Rieber N, Gazendam RP, Freeman AF, Hsu AP, Collar AL, Sugui JA, et al. Extrapulmonary Aspergillus infection in patients with CARD9 deficiency. JCI Insight. 2016;1(17):e89890.

4. Gavino C, Hamel N, Zeng JB, Legault C, Guiot MC, Chankowsky $\mathrm{J}$, et al. Impaired RASGRF1/ERK-mediated GM-CSF response characterizes CARD9 deficiency in French-Canadians. J Allergy Clin Immunol. 2016;137(4):1178-788.

5. Drummond R, Collar AL, Swamydas M, Rodriguez CA, Lim JK, Mendez LM, et al. CARD9-dependent neutrophil recruitment protects against fungal invasion of the central nervous system. PLoS Pathog. 2015;11(12):e1005293.

6. Gavino C, Cotter A, Lichtenstein D, Lejtenyi D, Fortin C, Legault $\mathrm{C}$, et al. CARD9 deficiency and spontaneous central nervous system candidiasis: complete clinical remission with GM-CSF therapy. Clin Infect Dis. 2014;59(1):81-4.

7. Drummond RA, Zahra FT, Natarajan M, Swamydas M, Hsu AP, Wheat LJ, et al. GM-CSF therapy in human caspase recruitment domain-containing protein 9 deficiency. J Allergy Clin Immunol. 2018;142(4):1334-8 e5.
8. Cuellar-Rodriguez J, Gea-Banacloche J, Freeman AF, Hsu AP, Zerbe CS, Calvo KR, et al. Successful allogeneic hematopoietic stem cell transplantation for GATA2 deficiency. Blood. 2011;118(13):3715-20.

9. Morillo-Gutierrez B, Beier R, Rao K, Burroughs L, Schulz A, Ewins AM, et al. Treosulfan-based conditioning for allogeneic HSCT in children with chronic granulomatous disease: a multicenter experience. Blood. 2016;128(3):440-8.

10. Cole T, Pearce MS, Cant AJ, Cale CM, Goldblatt D, Gennery AR. Clinical outcome in children with chronic granulomatous disease managed conservatively or with hematopoietic stem cell transplantation. J Allergy Clin Immunol. 2013;132(5):1150-5.

Publisher's Note Springer Nature remains neutral with regard to jurisdictional claims in published maps and institutional affiliations. 